\title{
1 Differential expression of potassium calcium-activated channel subfamily $M$ regulatory beta subunit 1 in human epithelial ovarian cancer.
}

Shahan Mamoor, MS ${ }^{1}$

${ }^{1}$ shahanmamoor@gmail.com

East Islip, NY USA

Epithelial ovarian cancer (EOC) is the most lethal gynecologic cancer (1). We performed discovery of genes associated with epithelial ovarian cancer and of the high-grade serous ovarian cancer (HGSC) subtype, using published and public microarray data $(2,3)$ to compare global gene expression profiles of normal ovary or fallopian tube with that of primary tumors from women diagnosed with epithelial ovarian cancer or HGSC. We identified the gene encoding potassium calcium-activated channel subfamily M regulatory beta subunit $1, \mathrm{KCNMB} 1$, as among the genes whose expression was most different in epithelial ovarian cancer as compared to the normal fallopian tube. KCNMB1 expression was significantly lower in high-grade serous ovarian tumors relative to normal fallopian tube. KCNMB1 expression correlated with progression-free survival in patients p53 mutant with ovarian cancer. These data indicate that expression of KCNMB1 is perturbed in epithelial ovarian cancers broadly and in ovarian cancers of the HGSC subtype. KCNMB1 may be relevant to pathways underlying ovarian cancer initiation (transformation) or progression.

Keywords: ovarian cancer, epithelial ovarian cancer, HGSC, high-grade serous ovarian cancer, systems biology of ovarian cancer, targeted therapeutics in ovarian cancer. 
The five-year survival rate for women diagnosed with high-grade serous ovarian cancer is between $30-40 \%$ and has not changed significantly in decades $(4,5)$. The development of novel, targeted therapeutics to treat HGSC can be facilitated by an enhanced understanding of the transcriptional behavior of ovarian tumors relative to that of the normal ovary. We mined published and public microarray data (2, 3) to compare global gene expression profiles between human ovarian tumors, including that of the HGSC subtype, and that of normal ovarian and fallopian tissue. We identified the gene encoding potassium calcium-activated channel subfamily $\mathrm{M}$ regulatory beta subunit 1, KCNMB1, as among the most differentially expressed in HGSC tumors of the ovary and in epithelial ovarian cancer broadly. KCNMB1 may be a gene of interest when prioritizing the study of target genes and pathways for the development of novel therapeutic interventions in epithelial ovarian cancer and specifically in high-grade serous ovarian cancers.

\section{Methods}

We used microarray data from datasets and GSE124766 (2) and GSE103737 (3) for this differential gene expression analysis of epithelial ovarian cancer in conjunction with GEO2R. The Benjamini and Hochberg method of p-value adjustment was used for ranking of differential expression but raw p-values were used for assessment of statistical significance of global differential expression. Log transformation of data was auto-detected, and the NCBI generated category of platform annotation was used. GSE124766 (2) was generated using Agilent-014850 Whole Human Genome Microarray 4x44K G4112F with $n=3$ normal fallopian tube tissue (control) and $n=7$ for tumors from patients with high-grade serous ovarian cancer; analysis was performed using platform GPL6480. GSE103737 (3) was generated using Illumina HumanHT-12 V4.0 expression beadchip with $n=17$ for stage 1 ovarian tumors and $n=72$ stage 3 and 4 ovarian tumors; analysis was performed using platform GPL10904. GEO2R provides mRNA expression levels only for the top 250 most differentially expressed genes transcriptome-wide.

A statistical test was performed to evaluate whether KCNMB1 expression was significantly different when comparing normal ovarian tissue from control subjects and primary tumors from women diagnosed with HGSC using a two-tailed, unpaired t-test with Welch's correction. We used PRISM for all statistical analyses (Version 8.4.0)(455). For Kaplan-Meier survival analysis, we used the Kaplan-Meier plotter online tool (6) for correlation of KCNMB1 mRNA expression levels with progression-free survival (PFS) in $n=483$ ovarian cancer patients.

\section{Results}

We mined published microarray data $(2,3)$ to identify differentially expressed genes in epithelial ovarian cancer and specifically in high-grade serous ovarian cancer (HGSC), the type of ovarian cancer responsible for $70-80 \%$ of deaths resulting from the most lethal gynecologic malignancy.

KCNMB1 is differentially expressed in ovarian tumors from women diagnosed with HGSC

We identified KCNMB1 as among the genes whose expression was most different when comparing primary HGSC tumors to normal fallopian tube tissue (2) (Chart 1). When sorting all of the transcripts measured by microarray based on change in expression between HGSC and the normal fallopian tube, in this data set, KCNMB1 ranked 34 out of 40481 total transcripts (Chart 1), equating to $99.9 \%$ differential expression. Differential expression of KCNMB1 in HGSC tumors was statistically significant (Chart 1; $p=1.48 \mathrm{E}-07)$. 
We analyzed a second microarray dataset (3) generated using normal ovarian tissues and tumors from women diagnosed with epithelial ovarian cancer (EOC) to determine whether differential expression of KCNMB1 could be observed in tumors from a different group of patients, which included HGSC tumors.

We found that KCNMB1 was also differentially expressed during the course of epithelial ovarian cancers broadly (Chart 2). When sorting all of the transcripts measured by microarray based on change in expression between tumors from patients at stage I and tumors from patients at stage III and IV, KCNMB1 ranked 4693 of 29088 total transcripts (Chart 2), equating to $83.9 \%$ differential expression. Differential expression of KCNMB1 in EOC based on disease progression was statistically significant (Chart 2; $\mathrm{p}=1.79 \mathrm{E}-02)$.

KCNMB1 is expressed at significantly lower levels in HGSC when compared to the normal fallopian tubes.

We then extracted exact mRNA expression levels for the differentially expressed KCNMB1 transcript from both normal fallopian tube tissue and from epithelial ovarian tumors. KCNMB1 was expressed at significantly lower levels in high-grade serous ovarian cancers when compared to the normal fallopian tubes (Figure $1 ; \mathrm{p}<0.0001$ ). We calculated a mean fold change of 0.59 in KCNMB1 expression when comparing HGSC tumors to normal fallopian tissue , as KCNMB1 was expressed at $12.95 \pm 0.24$ arbitrary units (AU) in the fallopian tubes but at $7.61 \pm 0.87 \mathrm{AU}$ in tumors of the ovary.

KCNMB1 tumor expression is correlated with survival outcomes in patients with ovarian cancer.

We performed Kaplan Meier survival analysis (6) to evaluate whether KCNMB1 tumor expression correlated with survival outcomes in patients with ovarian cancer. KCNMB1 primary tumor expression was correlated with progression-free survival (PFS) in patients with p53 mutant (p53 ${ }^{\text {mut }}$ ) ovarian cancer, in the upper tertile and in the upper quartile (Figure 2).

KCNMB1 expression was a negative prognostic indicator in patients with $\mathrm{p} 53^{\mathrm{mut}}$ ovarian cancer, in the upper tertile. While median PFS was 19.13 months for ovarian cancer patients with low tumor expression of KCNMB1, median PFS was 15.01 months for ovarian cancer patients with high tumor expression of KCNMB1 (Chart 3). Correlation of PFS with KCNMB1 tumor expression in patients with p53 ${ }^{\text {mut }}$ ovarian cancer, in the upper tertile, was statistically significant (Figure 2: log rank p-value: 0.00057; hazard ratio: 1.49 (1.19-1.87)).

KCNMB1 expression was a negative prognostic indicator in patients with $\mathrm{p} 53^{\text {mut }}$ ovarian cancer, in the upper quartile. While median PFS was 18.43 months for ovarian cancer patients with low tumor expression of KCNMB1, median PFS was 14 months for ovarian cancer patients with high tumor expression of KCNMB1 (Chart 3). Correlation of PFS with KCNMB1 tumor expression in patients with $\mathrm{p} 53^{\mathrm{mut}}$ ovarian cancer, in the upper quartile, was statistically significant (Figure 2: log rank p-value: 0.00033; hazard ratio: 1.55 (1.22-1.97)).

KCNMB1 primary tumor expression was not correlated with progression-free survival in the lower quartile (Figure 2: $\log$ rank p-value: 0.3 ; hazard ratio: $1.15(0.88-1.49)$ ) or in the lower tertile (Figure 2: log rank p-value: 0.14 ; hazard ratio: $1.2(0.94-1.52))$, in patients with $\mathrm{p} 53^{\mathrm{mut}}$ ovarian cancer. 


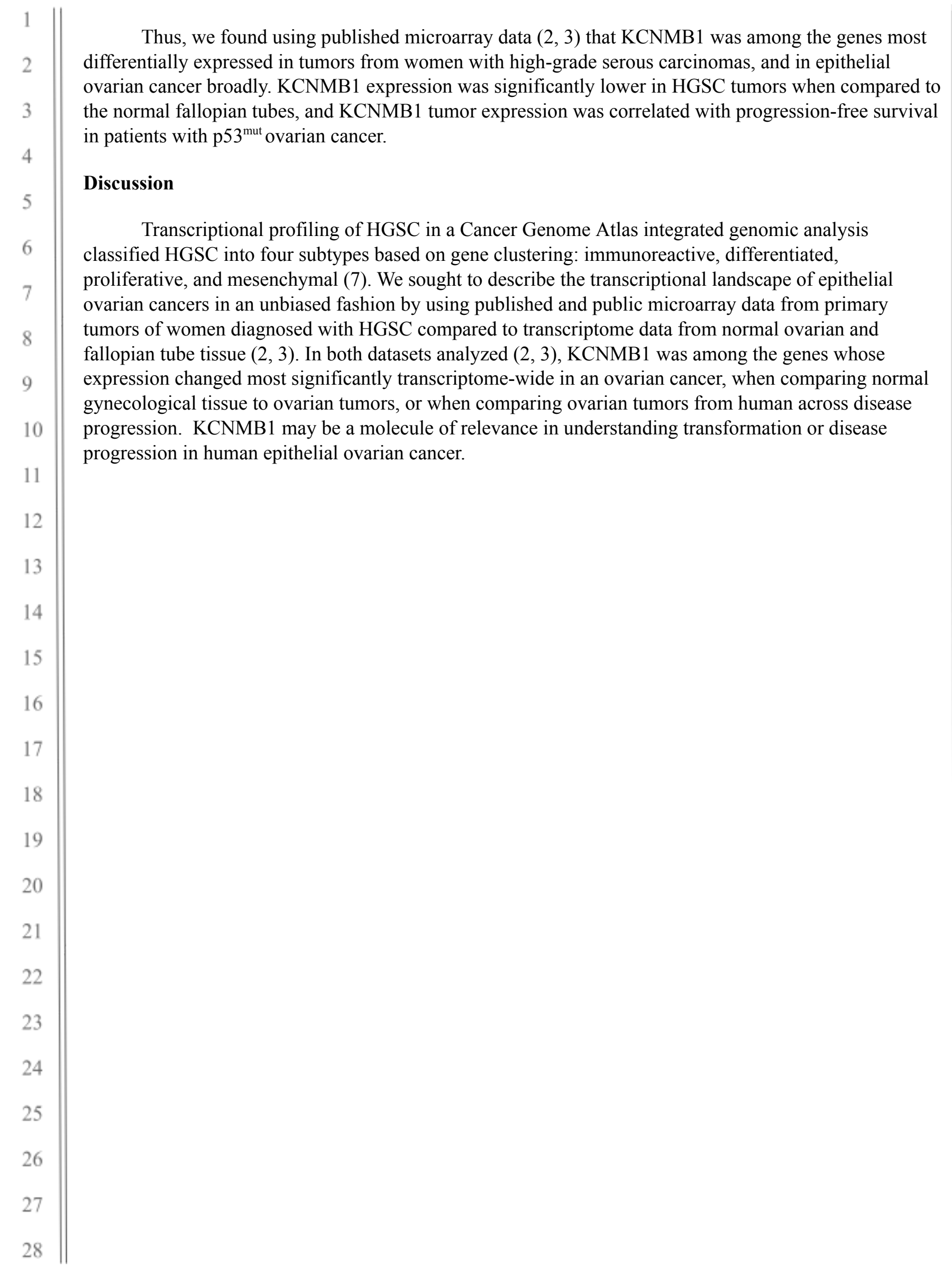




\section{References}

1. Guppy, A.E., Nathan, P.D. and Rustin, G.J., 2005. Epithelial ovarian cancer: a review of current management. Clinical Oncology, 17(6), pp.399-411.

2. Zhang, W., Klinkebiel, D., Barger, C.J., Pandey, S., Guda, C., Miller, A., Akers, S.N., Odunsi, K. and Karpf, A.R., 2020. Global DNA hypomethylation in epithelial ovarian cancer: passive demethylation and association with genomic instability. Cancers, 12(3), p.764.

3. Transcriptional correlates of high norepinephrine content in ovarian carcinomas. GSE103737. https://www.ncbi.nlm.nih.gov/geo/query/acc.cgi?acc=GSE103737. Steve Cole. UCLA School of Medicine.

4. Bowtell, D.D., Böhm, S., Ahmed, A.A., Aspuria, P.J., Bast Jr, R.C., Beral, V., Berek, J.S., Birrer, M.J., Blagden, S., Bookman, M.A. and Brenton, J.D., 2015. Rethinking ovarian cancer II: reducing mortality from high-grade serous ovarian cancer. Nature reviews Cancer, 15(11), pp.668-679.

5. Vaughan, S., Coward, J.I., Bast, R.C., Berchuck, A., Berek, J.S., Brenton, J.D., Coukos, G., Crum, C.C., recommendations for improving outcomes. Nature Reviews Cancer, 11(10), pp.719-725.

6. Győrffy, B., Lánczky, A. and Szállási, Z., 2012. Implementing an online tool for genomewide validation of survival-associated biomarkers in ovarian-cancer using microarray data from 1287 patients.

Endocrine-related cancer, 19(2), pp.197-208.

7. Cancer Genome Atlas Research Network, 2011. Integrated genomic analyses of ovarian carcinoma. Nature, 474(7353), p.609. 


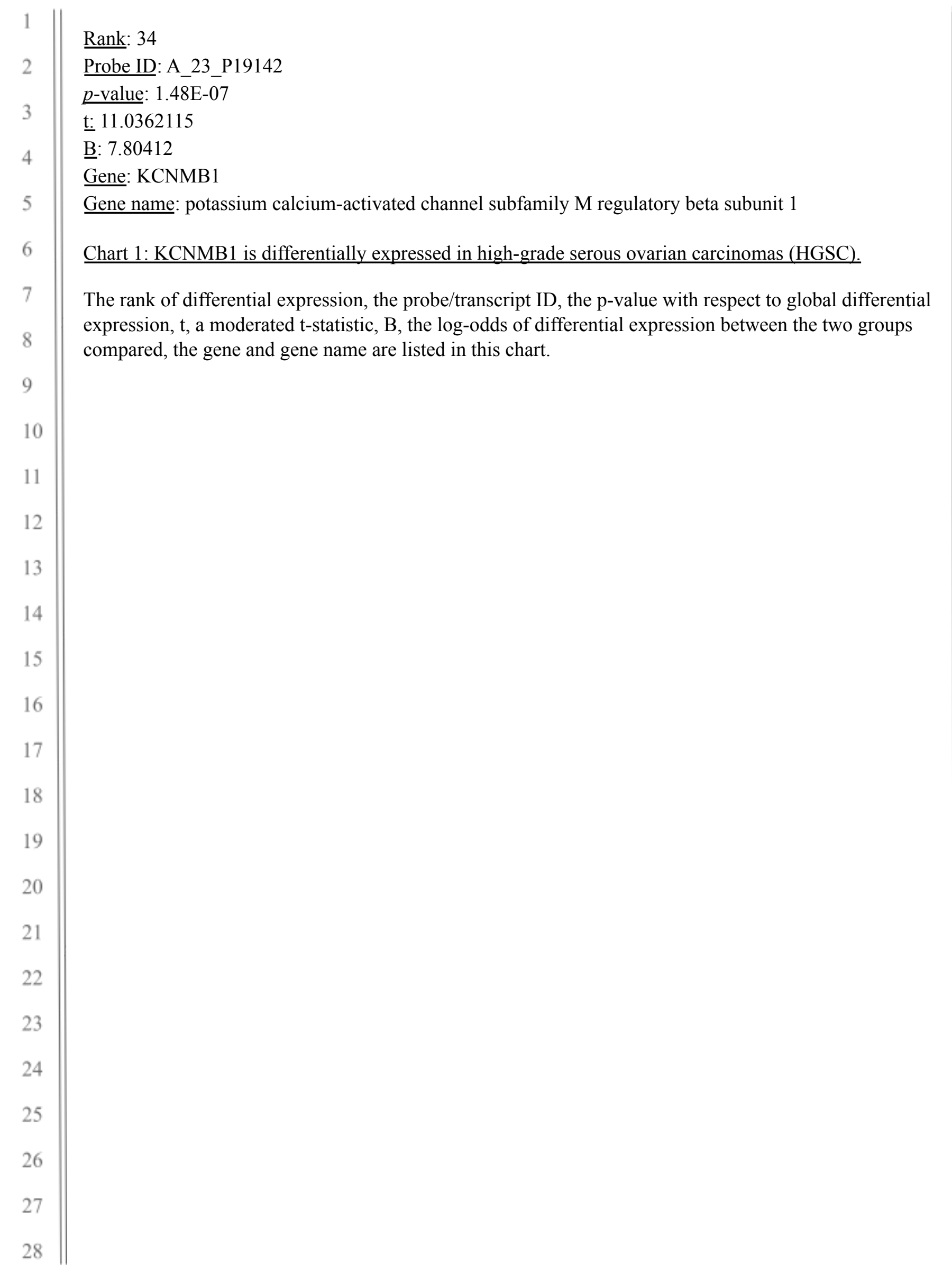




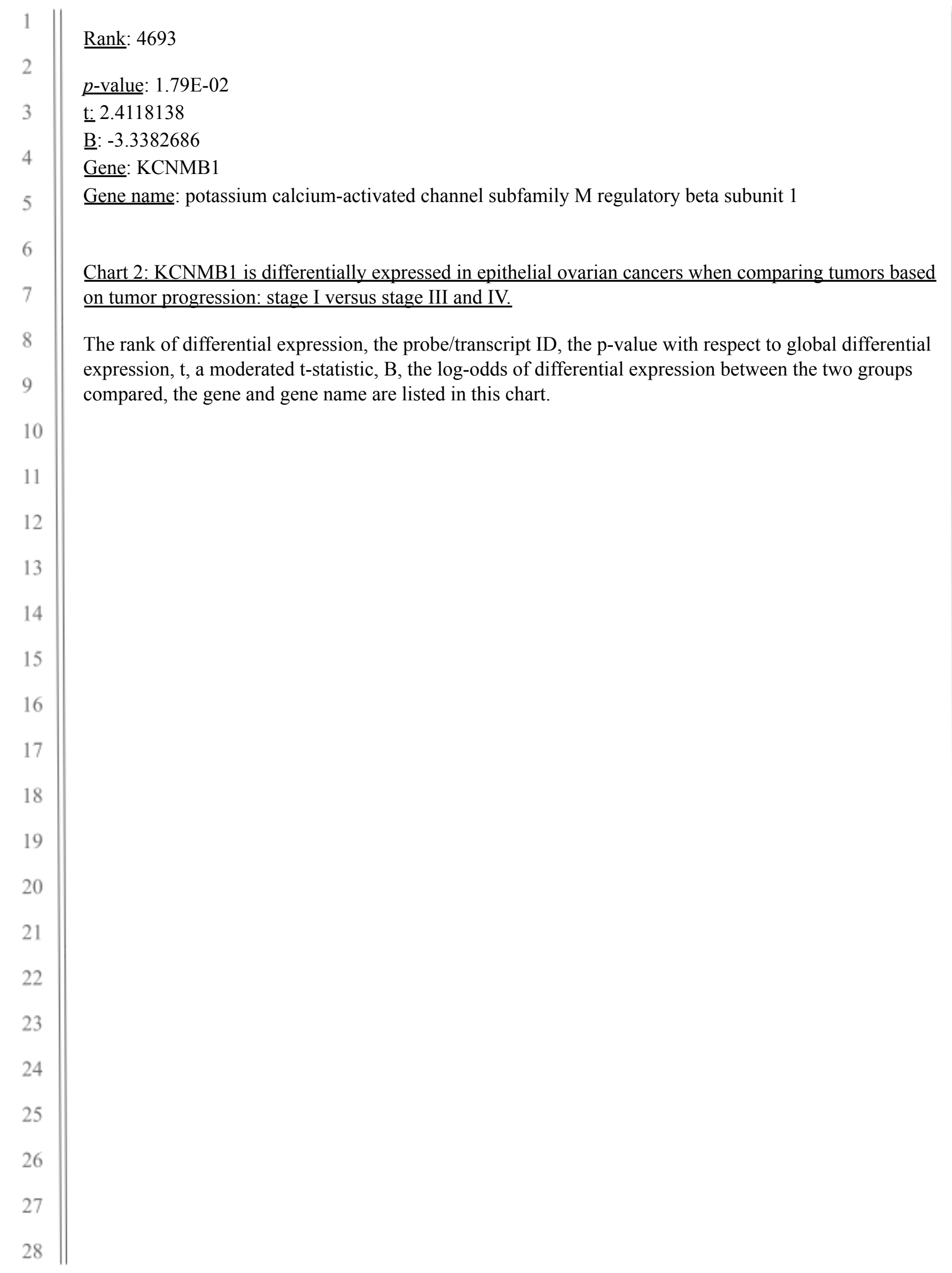




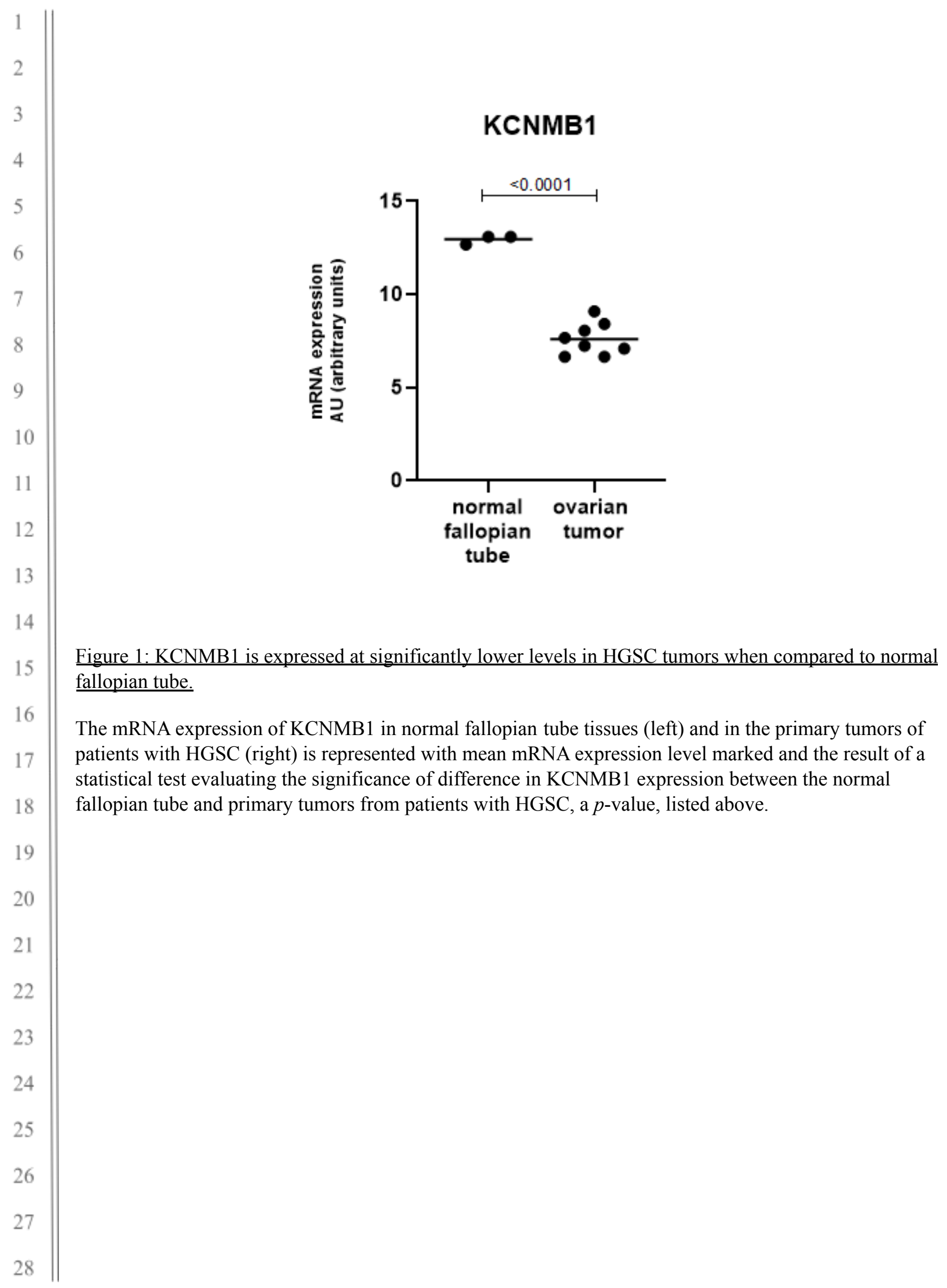



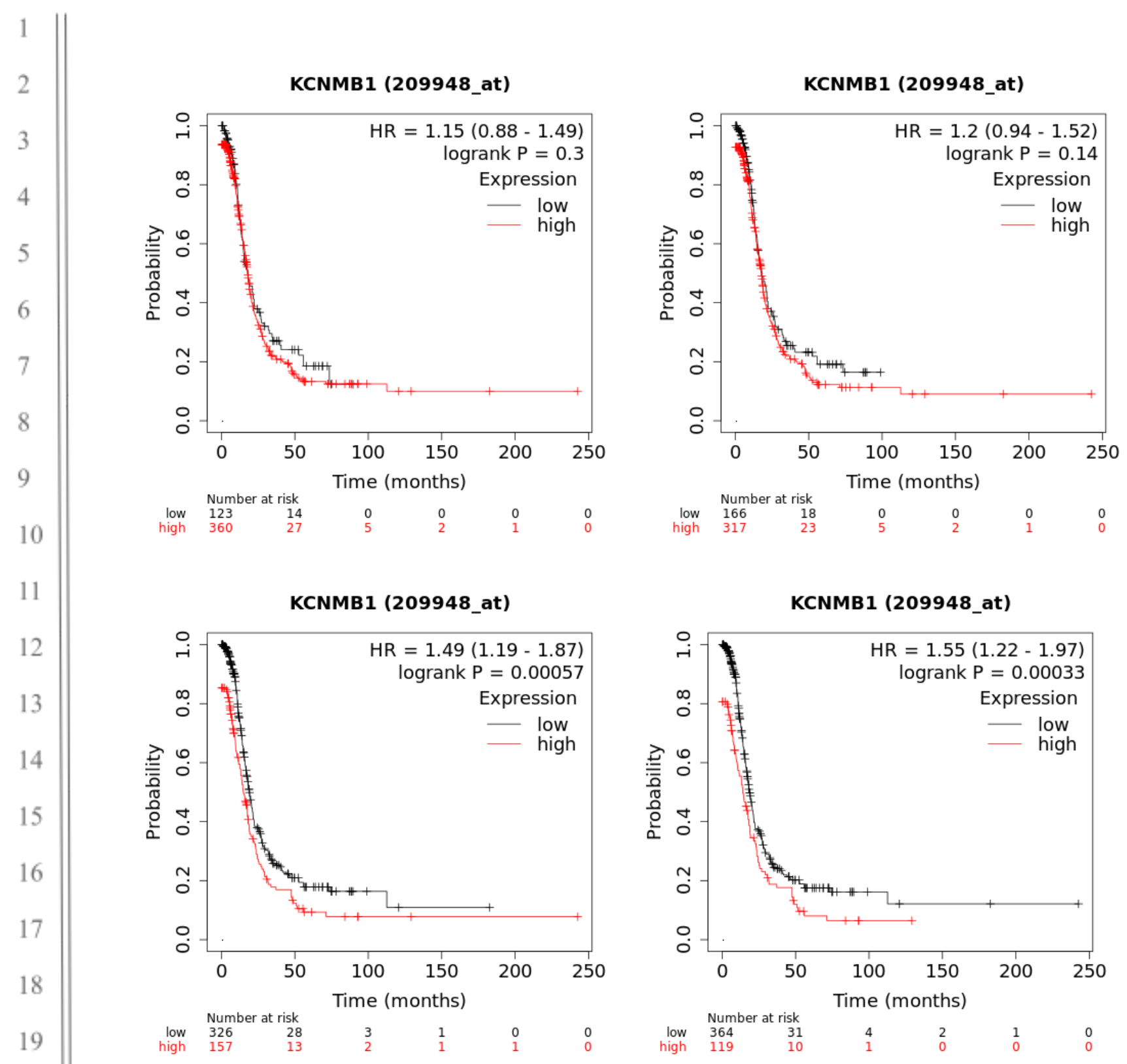

Figure 2: KCNMB1 tumor expression correlates with progression-free survival in patients with ovarian cancer.

Depicted in this Kaplan-Meier plot is the probability of progression-free survival (PFS) for $n=483$ patients, in the lower quartile (top left), in the lower tertile (top right), in the upper tertile (bottom left), and in the upper quartile (bottom right), based on primary tumor expression of KCNMB1 (low and high). The log rank $p$-value denoting statistical significance of difference in progression-free survival when comparing the two groups, as well as hazard ratio for this comparison is listed above. Listed below is the number of patients at risk (number of patients alive) per interval, after stratification based on KCNMB1 expression; in the first interval, number at risk is number of patients alive; in each subsequent interval, number at risk is the number at risk less those who have expired or are censored. 


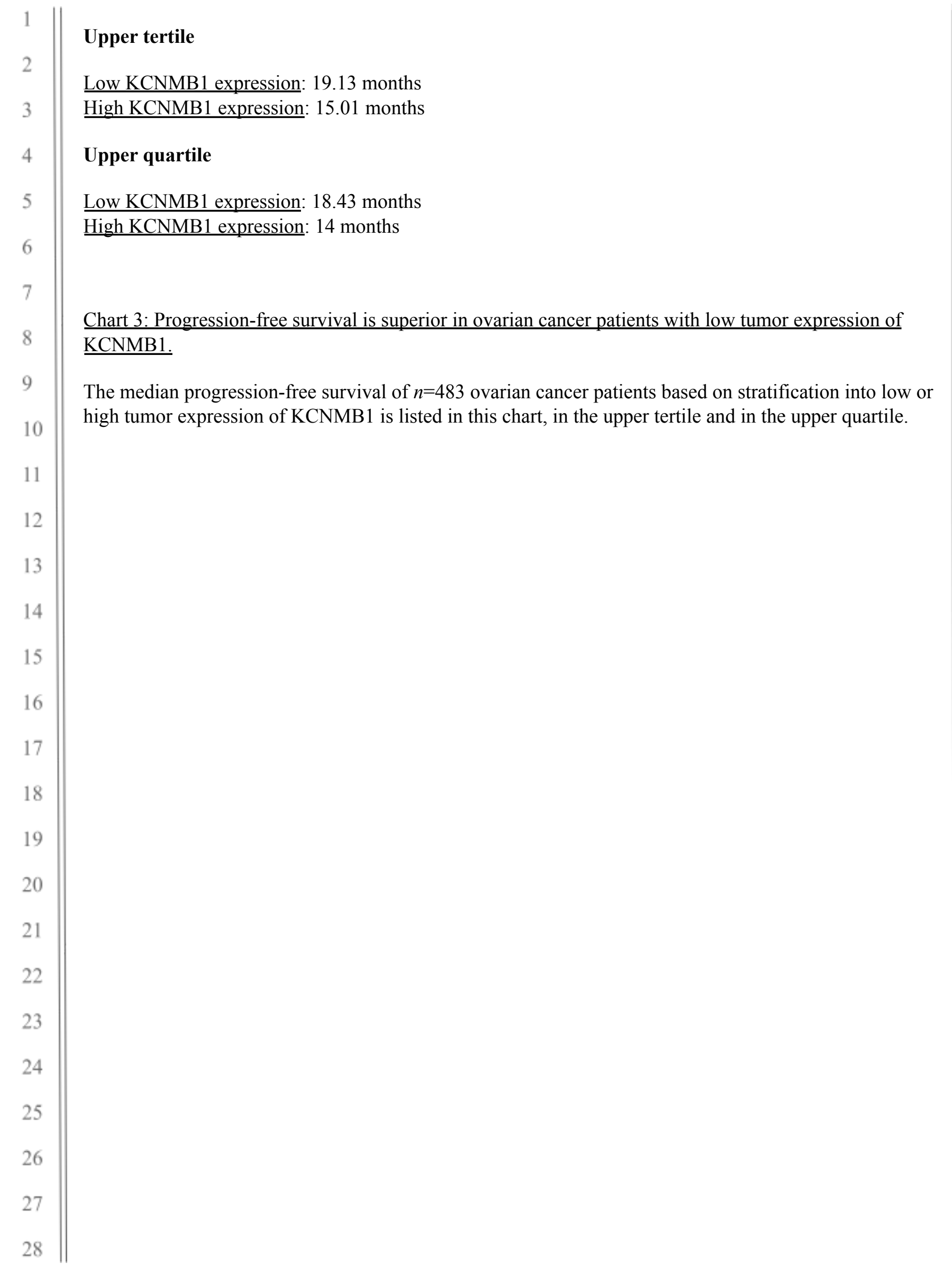

